\title{
TV/Series
}

3 | 2013

Écho et reprise dans les séries télévisées (I) : Reprise et intermédialité

\section{Cover, recover et uncover : les motifs de la reprise} dans la série Treme

\section{Peter Marquis}

\section{OpenEdition}

\section{Journals}

Édition électronique

URL : http://journals.openedition.org/tvseries/719

DOI : $10.4000 /$ tvseries. 719

ISSN : 2266-0909

Éditeur

GRIC - Groupe de recherche Identités et Cultures

Référence électronique

Peter Marquis, "Cover, recover et uncover : les motifs de la reprise dans la série Treme », TV/Series [En ligne], 3 | 2013, mis en ligne le 15 septembre 2013, consulté le 19 avril 2019. URL : http:// journals.openedition.org/tvseries/719; DOI : 10.4000/tvseries.719

\section{(c) (i) $\odot$}

TV/Series est mis à disposition selon les termes de la licence Creative Commons Attribution - Pas d'Utilisation Commerciale - Pas de Modification 4.0 International. 


\section{Cover, recover et uncover : les motifs de la reprise dans la série Treme}

Quoique Treme, le nouveau projet de Simon et Overmyer pour HBO, n'ait pas encore été diffusée dans son intégralité, un motif récurrent apparait, celui de la reprise. Il y a bien sûr la rénovation de La Nouvelle-Orléans après le passage de l'ouragan Katrina et de l'inondation qui lui fit suite, mais aussi la reconstruction de la culture locale, et celle des personnages. D'une manière ou d'une autre, ceux-ci sont tous engagés dans un processus de reconstruction, après des destructions de l'intime, du matériel, ou de l'immatériel. Cet article questionne la manière dont la série travaille le motif de la reprise, entendue comme répétition altérée d'un existant, aussi bien dans la vie des personnages que dans sa poétique même. Sur les onze épisodes de la saison 2, trois avatars de la reprise apparaissent : la reprise musicale (cover), la reprise métaphorique (recover) et la reprise critique (uncover). Ces trois motifs s'enchevêtrent pour donner à la série une tonalité idiosyncratique, mélange de lenteur, de compassion et d'indignation, une «note Treme » qui trouve son origine dans le recours généralisé à la figure de la reprise. En mettant la reprise au cœur de sa facture autant au niveau de la diégèse que de la métadiégèse, Treme ne cesse d'interroger les rapports complexes entre le pouvoir, la culture et la résilience des gens de peu.

$\mathrm{S}$ l'on retrouve dans Treme (HBO, 2010-) certains des thèmes favoris de l'auteur et producteur David Simon, tels la pauvreté urbaine, le racisme, ou la corruption, ainsi que quelques-uns de ses acteurs fétiches, cette nouvelle série n'est en rien une translation de The Wire (HBO, 2002-2008) à La Nouvelle-Orléans ${ }^{1}$. Son objet est la résilience de la culture ${ }^{2}$ et, plus précisément de la musique, à la fois fil rouge de la narration et moyen de subsistance des personnages. La série suit les vies de six musiciens : le tromboniste de soul music Antoine Batiste, le chanteur d'un collectif de Mardi Gras Indians ${ }^{3}$ Albert «Big Chief» Lambreaux, son fils le trompettiste de jazz Delmond Lambreaux, le compositeur de rap/funk Davis MacAlary, le guitariste de blues Sonny et la violoniste Annie Tee ${ }^{4}$. S'ils cherchent tous à vivre de leur musique, c'est surtout la musique qui les fait vivre,

${ }^{1}$ Cet article est la réélaboration d'une communication donnée à la journée d'étude «Transpositions, reprises, adaptations dans les séries télévisées américaines et européennes » organisée par l'ERIAC (Rouen) et le GRIC (Le Havre) à l'Université de Rouen le 11 juin 2012. Je tiens à remercier les organisateurs et les participants pour la pertinence de leurs remarques, ainsi que les différents relecteurs de cette version écrite.

${ }^{2}$ Résilience: "Propriété physique d'un matériau à retrouver sa forme après avoir été comprimé ou déformé » (Le Petit Robert).

${ }^{3}$ Les Mardi Gras Indians défilent lors des carnavals de La Nouvelle-Orléans en portant des costumes spectaculaires cousus par leurs soins et reprenant les motifs des habits de cérémonie des Amérindiens (plumes, perles, couvre-chefs). Leur origine remonterait à la moitié du $\mathrm{XVIII}^{\mathrm{e}}$ siècle. Les défilés s'accompagnent de chants qui ressemblent à des incantations où un meneur (le "Chief ») lance une phrase que les membres de la «tribu » reprennent en chœur. La rythmique est assurée par des percussions, des tambourins, etc. 4 A cette distribution musicale, il faut ajouter l'avocate Antoinette « Toni » Bernette, le policier Terry Coulson, la chef cuisinière Janette Desautel et le promoteur immobilier Nelson Hidalgo. 
ou plutôt survivre. En effet, Treme est une série du post-désastre qui se déroule intégralement dans l'espace-temps de La Nouvelle-Orléans quelques mois après la double catastrophe qui frappa la ville fin août 2005, l'ouragan Katrina et l'inondation qui lui succéda5.

Pourtant, rien ne serait plus faux que de voir dans Treme une œuvre à charge, une polémique qui aurait pour but de condamner les uns et de s'apitoyer sur les autres. La tonalité d'ensemble est d'ailleurs aux antipodes d'autres séries: l'action, quand il y en a une, est présentée aux téléspectateurs sans qu'ils en connaissent les enjeux ; les intrigues (cinq ou six en parallèle) avancent à petits pas, au sein de séquences inhabituellement courtes (une à deux minutes) et qui se font suite sans transition. Les personnages, sur lesquels les auteurs ne portent jamais un regard surplombant ${ }^{6}$, sont présentés dans leur complexité, saisis au cour de leur existence. C'est pourquoi la focale est souvent au raz des personnages : beaucoup de scènes sont tournées en gros plan, en intérieur, au milieu des foules de concerts ou de parades. Il résulte de ce positionnement que Treme est une série où on ne trouve ni morale ni pathos, mais bien plutôt réalisme et empathie.

Quoique la série n'ait pas encore atteint son terme, on peut déjà déceler un motif récurrent, celui de la reprise. Rebuild ou renew sont d'ailleurs des verbes fréquemment employés par les personnages qui, d'une manière ou d'une autre, sont tous engagés dans un processus de reconstruction, après des destructions de l'intime (divorce, viol, suicide, dépendance à la drogue), du matériel (perte de logement, dette) ou de l'immatériel (quête artistique, risque de perte d'une culture ancestrale)

Dans cet article, je souhaite questionner la manière dont la série travaille le motif de la reprise, entendue comme répétition altérée d'un existant, aussi bien dans la vie des personnages que dans sa poétique même. En analysant de manière thématique et narratologique les onze épisodes de la saison $\mathbf{2}^{7}$, trois avatars de la reprise apparaissent : la reprise musicale (cover), la reprise métaphorique (recover) et la reprise critique (uncover). L'enchevêtrement de ces trois formes fonctionne à plein dans l'économie circulaire si spécifique du genre sériel.

\footnotetext{
${ }^{5}$ Le début de chaque saison commence avec un carton de titre à la fois elliptique et hyperreférencé : "Three Months After »; "Fourteen Months After» et "Twenty-Five Months After ».

${ }^{6}$ Eric Overmyer, co-auteur, dira même pour justifier l'absence de didactisme: «Nous sommes avec les personnages ; nous ne savons que ce que les gens savent. » («We are with the characters, we know only whatever people know. »), " The Art of Treme », rencontre avec le public, Tulane University, 28 octobre 2010, Treme: The Complete Second Season, HBO, 2011. Traduction de l'auteur de l'article, comme les suivantes.

7 Le corpus ne retient que la saison 2 car la saison 1 a déjà fait l'objet de plusieurs études (voir bibliographie) et la saison 3 n'était pas encore disponible au moment de l'écriture du présent article.
} 


\section{Reprendre ou ne pas reprendre? L'ambiguïté des cover songs}

Contrairement à son emploi dans Law and Order (NBC, 19902010), ou dans les séquences finales de Cold Case (CBS, 2003-2010) ou de House, M.D. (Fox, 2004-2012), dans Treme, la musique ne sert pas de toile de fond émotionnelle à l'intrigue, mais joue un rôle narratif égal à celui des personnages. De nombreuses scènes sans dialogue lui sont consacrées, invitant les téléspectateurs à devenir, le temps de quelques minutes, auditeurs privilégiés d'un enregistrement ou d'un concert, le plus souvent enregistré live au moment du tournage. Parmi les morceaux entendus figurent un grand nombre de reprises, ce qui introduit une réflexion sur le rapport que les personnages entretiennent vis à vis de l'héritage et de l'original.

Parfois, le standard est encensé, si l'on en juge par les toutes premières images de la saison, qui montrent un jeune noir marchant dans les rues de son quartier le jour de la Toussaint (voir figure 1). Il apprend la trompette et exécute avec peine les premières notes de "When the Saints Go Marching In ", hymne non-officiel de la ville (2.1). Outre l'insertion d'un marqueur temporel important (la Toussaint) et le clin d'œil à Louis Armstrong, la présence, même fragile, de cet air si connu semble suggérer que la reprise des fondamentaux est nécessaire à toute reconstruction.

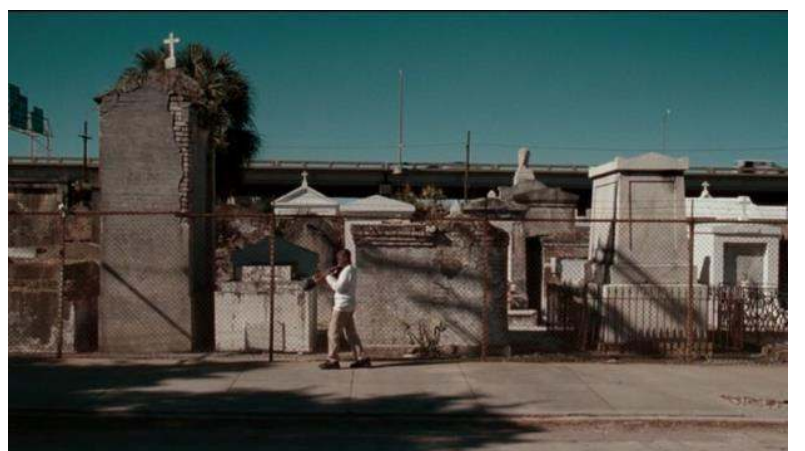

Fig. 1 : Jeune homme reprenant « When the Saints » le jour de la Toussaint (pré-générique, 2.1)

Placé dès l'incipit de la saison, ce message est d'autant plus fort qu'il est relayé à l'épisode suivant par la scène où " Del » Lambreaux, trompettiste de jazz contemporain vivant à New York mais né à La Nouvelle-Orléans, décide de jouer pour la station de radio qui l’invite le tout premier morceau que son père, musicien, lui avait enseigné dans sa jeunesse (2.2). 
L'héritage, s'il est présenté comme fondateur, n'est pas toujours simple à vivre : quelques épisodes plus tard, le même Del reprend à New York, devant un parterre d'amateurs de jazz d'avant-garde, un titre daté de 1923 du pionnier Jolly Roll Morton, intitulé « Milenberg Joys ». Après le concert, il confie à ses amis qu'il se sent gêné de l'avoir fait, comme s'il s'agissait d'une pulsion profonde, incontrôlable et presqu'inavouable (2.5). Cette reprise fait en réalité partie de la recherche que Del mène tout au long de la saison sur la création d'un style entre slave songs et free jazz.

Fondation plus ou moins bien assumée, la reprise peut être aussi envisagée comme un emprisonnement artistique inconscient ; la jeune violoniste Annie en fait cruellement l'expérience. Brillante musicienne d'accompagnement, elle est poussée par son ami Harvey à trouver sa voie en composant ses propres chansons. Après plusieurs jours de travail, elle présente le résultat à quelques amis qui la félicitent unanimement, avant qu'Harvey, plein de bienveillance pour sa protégée, ne raille : "Super! Tu as écrit un tube. Du moins, " Don't Think Twice » a bien marché pour Bobby Dylan ${ }^{8} \ldots$.. ». Il veut lui faire comprendre qu'elle a repris sans s'en rendre compte la grille d'accords de « Don't Think Twice, It's All Right » à une variation près. À l'épisode suivant, Annie, dépitée, en parle à son ami pianiste Tom McDermott, qui la rassure en lui expliquant qu'il a lui-même utilisé le thème d'un standard (« Heliotrope Bouquet » de Scott Joplin) pour composer l'un de ses morceaux (2.6). Cette résolution temporaire du dilemme portant sur l'originalité de toute création suggère que la pure création originale n'existe pas, autrement dit que tout est reprise, couche supplémentaire qui vient s'ajouter aux précédentes à demi effacées, tels les palimpsestes du Moyen-Âge.

Si Annie vit plutôt bien cette leçon d'humilité, Davis, lui, a un rapport plus problématique à la reprise comme filiation inévitable. Connaisseur érudit de la musique néo-orléanaise, c'est un musicien moins doué que les autres, qui vit cahin-caha grâce à ses piges de DJ et aux prêts d'argent de sa riche tante Minnie. On se souvient que, dans la saison 1, il a perdu son travail pour avoir refusé de diffuser lors de son émission à la radio les morceaux du patrimoine de La Nouvelle-Orléans au prétexte que, à ses yeux, la musique néo-orléanaise ne saurait être une collection de classiques rassemblés dans une compilation de Noël pour touristes (1.2). Même hantise de la nécrose culturelle dans la saison 2, quand Davis fonde son groupe, le politiquement nommé « DJ Davis and the Brassy Knoll9 » : soit celui-ci sera un mélange inédit de funk, jazz, rock, hip-hop et bounce, soit il ne sera pas (2.5). Son

\footnotetext{
${ }^{8}$ Harvey : « Nice, you wrote a hit [...] At least, "Don’t Think Twice" did alright for Bobby Dylan » (2.5)

9 Le nom fait référence au célèbre talus herbeux (grassy knoll) de Dallas, d'où, selon certains enquêteurs, le président Kennedy aurait été assassiné.
} 
exigence à " emmener», selon ses propres dires, «la musique néoorléanaise dans des contrées encore inconnues ${ }^{10}$ » le pousse même à négliger les conseils de ses amis qui ne croient guère en son projet, jugé trop hybride et militant.

Cet avis sévère semble être partagé par les scénaristes, à en juger par le sort qu'ils réservent au groupe de Davis : après des semaines passées à convaincre les stations de radio de diffuser des titres engagés politiquement, leur plus grand succès est un titre dansant aux paroles anodines composé non par Davis, mais par Calliope, le jeune rappeur nippo-américain recruté pour l'assister au chant. Prenant conscience de son échec en tant que meneur de groupe, Davis laisse sa place à un meilleur guitariste que lui et, pour son dernier concert, cède aux sirènes de la reprise, en s'essayant au « Sex Machine » de James Brown (2.11). Mais, avec beaucoup d'autodérision, il chante cette chanson en se glissant dans le rôle d'un blanc BCBG et coincé qui ânonne timidement les paroles au lieu de les adresser de manière fiévreuse (voir figure 2). Cet hilarant décalage, poursuivi sur plusieurs séquences, souligne que la reprise peut être tout autre chose qu'une abdication aux poncifs dès lors qu'elle est réappropriée par l'artiste.

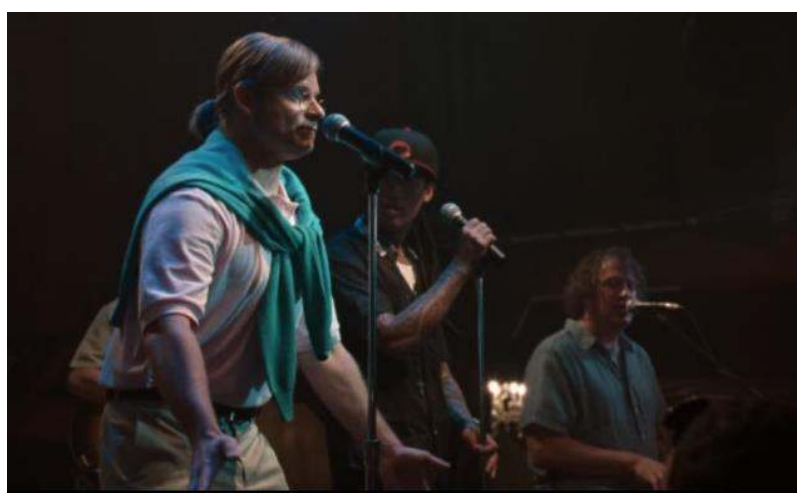

Fig. 2 : DJ Davis en James Brown BCBG (2.5)

Par conséquent, il n'est pas surprenant d'entendre dans Treme un nombre important de reprises qui sont avant tout des réappropriations ; on citera parmi d'autres les titres "Ac-Cent-TchuAte the Positive » (Arlen \& Mercer) repris par John Boutté en version acoustique, « Let's Stay Together » (Al Green) par "Antoine Batiste and the Soul Apostles » en version crooner, "Basin Street Blues » (Spencer Williams) en version piano-bar par Sonny, ou «Sex

${ }^{10}$ Davis : « I wanna bring New Orleans music to yet uncharted territories » (2.5) 
Machine » (James Brown) en version... fils de bonne famille. Dans ce temple du jazz qu'est La Nouvelle-Orléans, l'original compte donc moins que la reprise, cette touche unique que chaque musicien apporte (ou dérobe ?11) au canon. La leçon s'apprend dès le plus jeune âge : Antoine, devenu enseignant à mi-temps pour une fanfare scolaire, fait écouter un morceau de Louis Armstrong à la classe ; un jeune, guère impressionné, commente négligemment: « Je peux le jouer note à note demain »; Antoine répond: «D'accord, mais peux-tu le jouer à ta façon demain? Et à ta façon, mais de manière différente, tous les autres jours? ${ }^{12}$.

L'accent mis sur la réappropriation résout le dilemme de la pure création en la présentant comme produit simultané de la liberté et de la contrainte. Cette vision est thématisée par la recherche artistique du trompettiste Del, qui finit par trouver le point de conciliation entre la tradition et la nouveauté en fusionnant, dans un seul groupe, le son des Mardi Gras Indians de son père et le jazz contemporain qu'il pratique (2.11). L'album est un succès critique, mais il est intéressant de remarquer que l'alchimie entre les musiciens n'a pu fonctionner qu'à La Nouvelle-Orléans (et non à New York) comme si, dans toute création, le poids de l'héritage (la reprise) était toujours plus fort que celui du présent (la nouveauté).

On comprend donc qu'ici, la reprise musicale est bien une cover, une version secondaire qui vient couvrir une version initiale, sans toutefois la recouvrir complètement, rendant sensible à tous l'origine de toute création, ou du moins sa trace. Cela fait écho aux propos de David Simon qui, interrogé sur le risque de fossilisation de la musique de La Nouvelle-Orléans et donc du rôle de sa série dans un processus de nécrose culturelle, avait répondu : "Certains musiciens après Katrina ont pu chercher un son fossilisé, mais la majorité appartient à une scène qui ne cesse de se transformer ${ }^{13}$ ». Dans la poétique de David Simon, la même chose pourrait être dite des personnages, qui traversent tous une période de reprise, à l'image de leur ville.

\section{La reprise au sein de la narration : recovery et ses affres}

Après Katrina et les inondations qui suivirent, La NouvelleOrléans entama une phase de reconstruction. Bon an mal an, celle-ci fut entreprise par de multiples acteurs - aussi bien par l'État fédéral (FEMA; Army Corps of Engineers), que par la Ville (Bring New

\footnotetext{
${ }^{11}$ En anglais, ne dirait-on pas « a musician's take on a song » ? (je souligne)

12 «I can play it note for note tomorrow »; «Alright, but can you play it your way tomorrow, and your way but in a different way every other day? » (2.6)

13 "Some musicians after Katrina may have been looking for a sound caught in amber, but the majority is part of a scene that is constantly transforming itself », Simon, " The Art of Treme », op. cit.
} 
Orleans Back Commission), la Croix Rouge, les églises, ou les associations comme Habitat for Humanity. Pour de multiples raisons, la reconstruction fut lente et génératrice de frustrations pour beaucoup de citoyens, notamment les plus pauvres ${ }^{14}$. S'ils sont palpables en arrière-fond, ces aspects du temps historique ne constituent pas le cœur du temps diégétique de Treme. La question de la reconstruction (ou recovery) est toujours abordée par petites touches et, surtout, par le biais des personnages qui vivent et parfois incarnent cette période de reconstruction.

Alors qu'on pourrait s'attendre à ce que le besoin de reprise des personnages provienne des effets de l'ouragan Katrina (inondation, dettes, décès), cette catastrophe est plutôt présentée comme un accélérateur d'infortunes qui, paradoxalement, permet parfois la possibilité de renouveau pour certains personnages. Si quelques-uns s'approchent de leur objectif, métaphorisé dans l'expression come home (retourner chez soi, se sentir à sa place), d'autres peinent. Comme souvent dans l'art de David Simon, le ton n'est pas triomphaliste : aucun des personnages ne sort de la saison 2 en ayant complètement accompli sa quête. Ainsi, jamais la reprise, entendue comme recovery, n'est-elle totale ou sans nuance, mais elle est toujours présentée comme une incomplétude fondamentale, un effort de résistance, voire une résilience.

Rappelons que le quartier Tremé/Lafitte auquel la série doit son nom se situe sur les hauteurs de La Nouvelle-Orléans et n'a donc pas subi de dégâts directement causés par l'inondation, contrairement au Lower Ninth Ward, par exemple ${ }^{15}$. Cependant, dès le générique, Katrina et ses effets sont bel et bien présents à travers des images de tourbillons d'eau, de rues inondées (voir figure 3) et de murs moisis.

14 Voir Hugo Lefrebvre, Enjeux de la reconstruction de la Nouvelle-Orléans après l'ouragan Katrina, Editions L'Harmattan, 2008 ; Karl F. Seidman, Coming Home to New Orleans: Neighborhood Rebuilding After Katrina, Oxford University Press, 2013.

${ }_{15}$ Michael Eugene Crutcher, Tremé: Race and Place in a New Orleans Neighborhood, Athens (GA), University of Georgia Press, 2010, p. 116. 


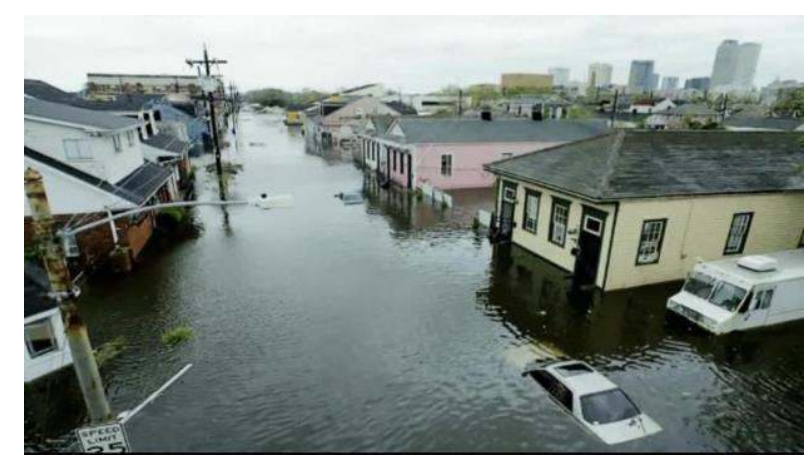

Fig. 3 : Une rue sous les eaux après Katrina (image d'archives, générique)

Si le thème de la destruction domine le générique, celui de la reconstruction de la ville, lui, n'est abordé dans la série que par la négative, à savoir la lenteur et l'inefficacité avec lesquelles les autorités ont pris en charge le retour des 800 ooo réfugiés et la rénovation des 200 ooo habitations endommagées. Dans une scène $a$ priori anodine, toute la tragédie de cette période est mise en scène. Albert Lambreaux, son fils Del et un ami artisan sortent d'un magasin de bricolage, le caddie plein d'ustensiles. Ce magasin, bien réel ${ }^{16}$, appelé "The Preservation Salvage Store », est en fait un dépôt-vente qui met à disposition des clients des matériaux récupérés sur des maisons détruites par l'ouragan et l'inondation (2.2). Le dialogue ne nous apprend rien de cela, mais seulement qu'Al doit recommencer les réparations qu'il a effectuées pour la plomberie de sa maison car les autorités sanitaires n'ont pas validé ses travaux. La reconstruction semble donc rendue impossible du fait d'un appareil bureaucratique écrasant, et ce malgré les efforts des citoyens à s'en sortir par euxmêmes.

Il en va de même pour la reconstruction, non pas des murs et des tuyaux, mais de la culture et des êtres. Le quotidien d'Albert Lambreaux a été bouleversé par Katrina, car il a perdu le local où il avait l'habitude de faire vivre un élément central de la culture néoorléanaise: c'est là qu'il répétait avec son groupe de Mardi Gras Indians et qu'il cousait les fameux costumes utilisés lors des parades traditionnelles. Dans l'intérim, il occupe un bar laissé vacant, avant de s'en faire expulser par le propriétaire (2.5); pis, la plupart de ses acolytes vivent toujours en exil dans le nord de la Louisiane ou au Texas, n'ayant pas pu être relogés, ce qui est un frein majeur à la reconstruction de leur groupe de Mardi Gras Indians. Al est donc contraint de reprendre un travail de plâtrier dans une entreprise de

${ }^{16}$ Voir le site web http://www.prcno.org/shop/salvagestore.php, consulté le 16 juillet 2013. 
rénovation de maisons bourgeoises dans les beaux quartiers (2.2) (voir figure 4). Ironie du sort, ces maisons n'ont pas été touchées par Katrina. C'est donc celui qui souhaite se reconstruire et reconstruire la culture locale qui se voit contraint de rebâtir non pour lui, mais pour des personnes pour qui ce n'est pas une nécessité impérieuse.

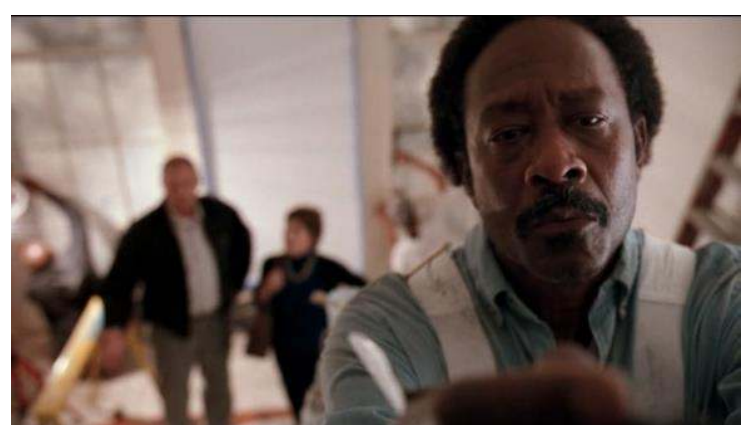

Fig. 4 : Al en train de rénover une corniche, sous les yeux de propriétaires aisés (pré-générique, 2.2)

Tout aussi problématique est la reconstruction de soi, comme l'illustre le cas de Davis. Pour plaire à sa nouvelle petite amie, la violoniste Annie, il décide de se reprendre en main et de nettoyer sa garçonnière d'ex-célibataire désordonné. Il finit par se décourager et jette à la poubelle tout ce qu'il ne peut pas ou ne veut pas laver ni ranger (2.1) (voir figure 5). Malgré le comique de la scène, on peut y lire beaucoup de sarcasme de la part de l'auteur : n'est-ce pas là le reflet en miniature de ce qui se passe à l'échelle de la ville ? Faut-il reconstruire à partir de l'ancien, ou tout raser et repartir à zéro ?

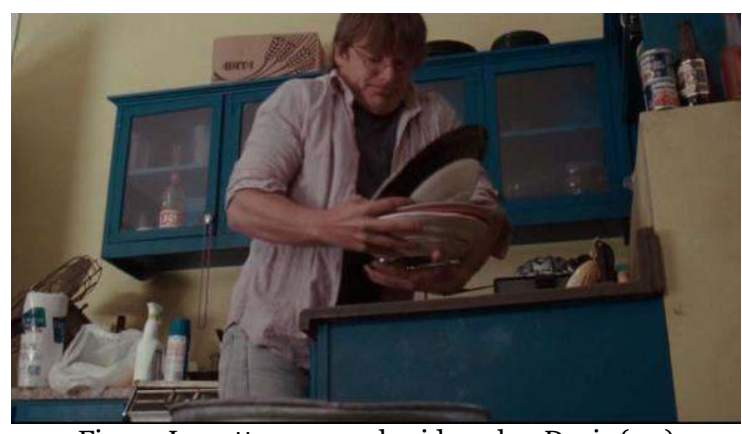

Fig. 5 : Le nettoyage par le vide, selon Davis (2.1)

Cette question est au cœur du parcours d'Antoine Batiste, tromboniste accompli qui, après des années à vivoter de concert en 
concert, se dit «à un carrefour de sa vie ${ }^{17}$ ». Il abandonne ses engagements tous azimuts et rassemble des musiciens pour créer son propre groupe. Mais celui-ci ne perce pas car il se compose de pièces rapportées engagées par ailleurs et qui n'ont guère d'ambition sinon de faire bouillir la marmite. À la fin de la saison, Antoine doit donc dissoudre le groupe, ce qui laisse à entendre que, s'agissant de lui, chercher à mener et à briller n'était pas la bonne solution. Au contraire, il semble trouver un certain salut en dirigeant une fanfare de rue composée de jeunes gens, dont certains sont des élèves de l'école où il est professeur de musique assistant. Parmi eux se trouve l'apprenti trompettiste vu dans les premières scènes de la saison. Cette reprise est double : le jeune homme rappelle à Antoine ses débuts de musicien, et aux spectateurs les scènes de l'incipit, creusant le sillon de la reprise comme motif central de Treme tant au niveau de la diégèse que de la métadiégèse.

En effet, dans Treme, la reprise entendue comme recovery est inséparable d'un regard tourné vers le passé et d'un rapport douloureux à la filiation. Cette question hante le trompettiste Del Lambreaux : quand, à la toute fin de la saison, il parvient à marier la tradition de son père avec la modernité qui est la sienne, il doit en payer le prix. Sa petite amie new-yorkaise le quitte car il retourne habiter à La Nouvelle-Orléans pour enregistrer avec son père, l'aider à finir sa maison et, surtout, devenir un Mardi Gras Indian, c'est-à-dire maîtriser l'art du beadwork (2.11)

La chef Janette, elle aussi, veut se reprendre en main après la fermeture de son restaurant à La Nouvelle-Orléans et sa rupture avec Davis. Elle s'exile à New York quelques mois, y rencontre un franc succès en tant que chef dans des établissements cotés de la ville. Elle est alors contactée par un agent culinaire qui lui propose d'investir avec elle pour ouvrir son restaurant à La Nouvelle-Orléans. Elle finit par accepter, retourner " chez elle », mais perd en contrepartie son poste à New York. Elle aussi doit payer un prix sentimental : sa solide relation professionnelle avec son sous-chef Jacques est détruite à jamais, selon elle, dès lors qu'ils ont eu une relation sexuelle ${ }^{18}$.

Si regarder en arrière peut parfois permettre un nouveau départ (quoique jamais gratuit), c'est pour certains personnages une spirale d'autodestruction. Tel est le cas du guitariste Sonny qui, séparé d'Annie, replonge dans la consommation de drogue dure (1.10). Le salut que lui accordent les auteurs ne vient pas du retour aux origines, mais du déplacement : il se désintoxique en partant travailler plusieurs semaines sur un petit bateau de pêche avec d'autres compagnons

\footnotetext{
17 « I feel I'm at a crossroads in my life. » (2.3)

18 Lors de sa première soirée, bien arrosée, à La Nouvelle-Orléans, elle succombe aux charmes de son sous-chef antillais ; or, la règle d'or du métier exige de ne jamais coucher avec son personnel car « un sous-chef, c'est pour la vie » (en français dans le texte) (2.11).
} 
contraints au sevrage, sous la férule intransigeante de leur employeur vietnamien (2.7).

La toxicité du passé plane également sur Antoine Batiste, pour qui la filiation est un sujet générateur de regrets. Au contact de ses élèves musiciens, il se rend compte trop tardivement qu'il a négligé ses propres enfants, dont la garde revenait à son ex-épouse LaDonna. Il s'en veut de ne pas avoir fait de ses deux fils des musiciens, comme son père et son grand-père l'avaient fait pour lui : « Je n'ai pas été à la hauteur de leurs attentes ${ }^{19}$ » se lamente-t-il. Tout laisse à croire qu'à ses yeux, ne pas transmettre revient à commettre un crime d'hubris, où l'arrogance consiste à ne pas occuper sa juste place dans cette communauté de musiciens. Ne pas assurer la transmission du flambeau - autrement dit, ne pas garantir la reprise - revient à se condamner à une vie de regrets, lexicalisée par l'expression «j'aurais dû le voir venir» («I shoulda seen it comin'»), prononcée par plusieurs personnages dans la série.

Enfin, Treme aborde le thème de la reprise sous l'angle du retour à la vie après l'expérience de la mort, thème qui plane sur la saison 2 dès son premier épisode dans la mesure où, en ce jour de la Toussaint, tous ont quelqu'un ou quelque chose dont ils doivent faire le deuil. Pour Toni et Sofia Bernette, le deuil du mari et du père (Creighton Bernette, qui s'est suicidé dans la saison 1) passe par le fait de renouer avec les habitudes de l'être disparu. Par exemple, quand le marchand de glaces italien rouvre ses portes, Sofia et Toni se régalent de la lemon ice que Creig adorait. Le deuil prend aussi une forme plus individuelle, lorsque Sofia reprend mot pour mot les insultes que son père avait formulées contre l'État fédéral dans un billet vidéo posté sur YouTube au tout premier épisode de la série ${ }^{20}$; de même, en 2.7 Toni se rend sur les berges pour jeter dans le fleuve quelques effets de son mari. La reprise, qui jusque-là avait été au service de l'hommage ou de la résilience, se mue en figure du souvenir des disparus.

Pour le personnage de LaDonna, la reprise sert de support à la colère. Tenancière d'un bar de nuit dans le quartier Tremé, elle est tabassée et violée un soir alors qu'elle ferme son établissement (2.3). Cachant cette tragédie à son mari, elle glisse dans la dépression, s'exile à Bâton-Rouge, s'isole dans sa chambre et tente de noyer sa colère dans le whisky. Elle sort de sa torpeur en voyant un jour son agresseur, qui avait pourtant été interpellé par la police, assis dans un bar à boire une bière avec des amis. Elle appelle la police, finit par le frapper ellemême devant les policiers, puis elle se rend au commissariat et livre une diatribe contre les services de police :

19 « I failed them » (1.8).

${ }_{20}$ « This so-called recovery sucks, nothing's getting fixed; and the National Guard is patrolling our streets like we're fucking Fallujah! Fucking fucks! » (2.1) (« cette soit-disant reprise craint, on ne répare rien ici, et la Garde nationale patrouille dans nos rues comme si on était à Fallujah ; putains de gros cons ! »). 
Mais putain, qu'est-ce qui déconne dans vos services ? Vous perdez mon frère dans une putain de prison pendant des mois et maintenant, à la première occasion, vous laissez filer ce petit enfoiré... (pause) On essaie de vivre dans cette ville, on essaie de revenir ici, de remettre de l'ordre dans le peu de choses qu'on possède, de vivre; et tout ce que vous trouvez à faire, c'est que dalle (soupir méprisant) Construire une maison? Sûrement pas. Remplis ce formulaire et patiente. Remettre les gosses à l'école? Laquelle? Vous avez trois systèmes scolaires différents, dont deux qui enseignent que dalle [...]. Rouvrir un hôpital ? Sûrement pas. Plutôt détruire quelques quartiers en plus. Coffrer un criminel ou deux ? Plutôt crever [...] $]^{21}$.

La colère de LaDonna glisse donc du personnel au collectif tant cette diatribe fustige toute la politique municipale et fédérale de prétendue reprise. En effet, si Treme est une série de la recovery, elle s'évertue aussi à reprendre des faits réels et à les exposer à la critique (uncover).

\section{La reprise extradiégétique : uncovering}

Quels rapports la série entretient-elle avec le contexte historique dont elle tire son matériau, à savoir La Nouvelle-Orléans de l'après-Katrina ? À y regarder de près, l'ouragan et l'inondation qui l'a suivi ne figurent pas exactement au centre de l'œuvre ; cette place est occupée par la ville elle-même et sa culture, aussi bien musicale que gastronomique. La reprise ici s'apparente en fait à la reconstitution du réel d'une petite communauté dans un souci d'authenticité ${ }^{22}$ et d'une forme de compassion pour la résilience de ces personnages. Plus que la ville, c'est un quartier qui est reproduit. Le quartier de Tremé ${ }^{3}$ est le quartier noir historique de La Nouvelle-Orléans, berceau de la musique afro-américaine (les noirs affranchis y jouaient au XVIII ${ }^{\mathrm{e}}$ siècle, notamment sur Congo Square). Aujourd'hui, c'est le lieu de vie de nombreux artistes, et le point de départ de défilés de fanfares (brass

\footnotetext{
${ }^{21}$ « What the fuck is wrong with you people? You lose my brother in a damned jail for one, and then you let this vicious motherfucker go the first chance you get... we tryin' to live in this city, we tryin' to come back here, put what little shit we got back together and live; and all you manage to bring to that is nothing (sniggers). Build a house? Hell no! Fill out this form and wait. Get your child back in school? Which one? You got three different school systems, two of them ain't teaching shit [...]. Open a hospital back up? Hell, no! Let's tear down some more neighborhoods instead. Solve a crime or two? Oh hell the fuck no! » (2.11). La deuxième phrase se réfère en particulier au fait que son frère a été abattu dans des circonstances étranges dans les jours qui ont suivi l'ouragan, sans que la police ne mène de réelle enquête.

${ }^{22}$ Sur l'« authenticité » dans Treme et les problème que ce terme pose, voir l'émission en balado-diffusion "The Cultural Gabfest - Ooh La La edition », 14 avril 2010, http://www.slate.com/articles/podcasts/culturegabfest/2010/04/the culture gabfest oo h la la edition.html, consulté le 16 juillet 2013.

${ }^{23}$ La City Planning Commission circonscrit le quartier Tremé de la sorte : Esplanade Avenue à l'est, North Rampart Street au sud, St. Louis Street à l'ouest et North Broad Street
} au nord. 
bands et second lines) organisés par les associations de quartier (krewes), ainsi que d'hommages musicaux aux défunts (jazz funerals). Le générique utilise d'ailleurs des images d'archives de ces manifestations culturelles (voir figure 6).

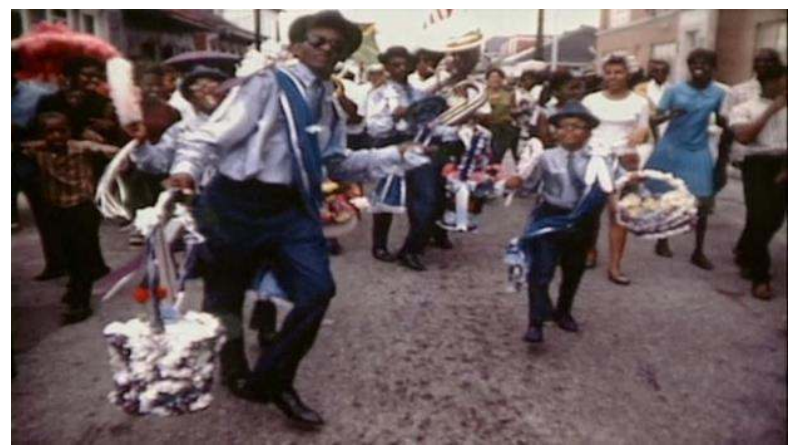

Fig. 6 : Image d'archives d’une second line (générique)

Plus généralement, Treme utilise les toponymes réels des quartiers, des rues, des restaurants (Mosca's, Dooky Chase) et des clubs (Blue Nile, House of Blues, Spotted Cat, etc.). Le souci d'authenticité va même jusqu'à faire jouer leur propre rôle à des personnages réels, comme Oliver Thomas, président du Conseil municipal, pressenti pour devenir maire, qui fut incarcéré pour malversation en 2007 (son arrestation est intégrée à l'épisode 2.11). Les téléspectateurs suivent également la vie des musiciens réels que sont Kermit Rufins, Dr. John, Tom McDermott, le groupe Galactic, ou l'étoile montante du jazz néo-orléanais « Trombone » Shorty.

S'ajoutent à ces musiciens la chef Susan Spicer (qui fait une apparition dans la saison 2), ou les propriétaires du restaurant Bacchanals, qui ouvrirent leurs fourneaux à des chefs mis au chômage technique par Katrina (1.9). De plus, les moments forts de la vie néoorléanaise sont représentés, comme la fête de Mardi-Gras (un épisode entier lui est consacré en 2.7) ou le « Jazz Fest » (2.11). L'ambition n'est pas tant de donner un effet de réel, que de reconstituer la culture d'une ville à un moment donné de son histoire, dans un souci de fidélité factuelle et émotionnelle. Par exemple, le rôle de Desiree, la compagne d'Antoine Batiste, fut donné à une survivante de Katrina, qui n'avait aucune expérience de comédienne. La fiction épouse donc la réalité, parfois pour mieux en critiquer l'injustice.

En effet, Treme peut aussi être regardée comme une série à charge contre l'inaction de l'État fédéral et du maire Ray Nagin face au désarroi des populations touchées par l'inondation résultant de l'ouragan. Dès le générique, le ton est donné : celui-ci, quoique posé sur une musique entraînante, est truffé d'images d'archives qui mettent 
l'accent sur la destruction, la désolation, la dépossession, la colère, mais aussi la tradition et l'humour ${ }^{24}$ (voir figure 7).

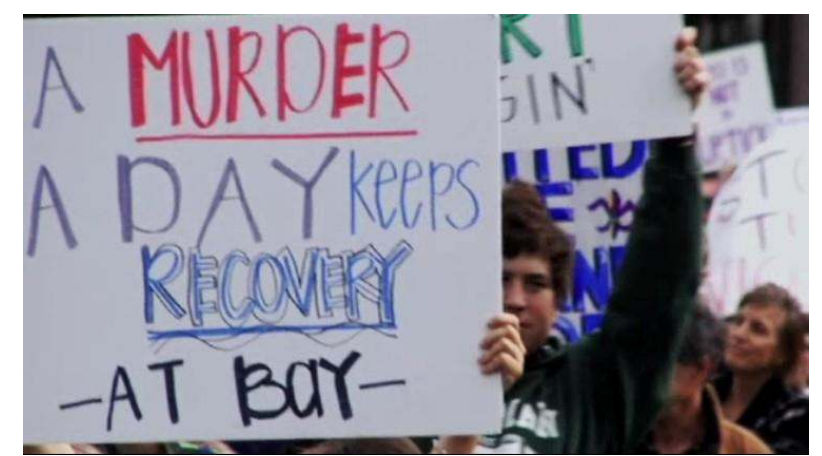

Fig. 7 : Image d’archives des manifestations post-Katrina (générique)

Treme convoque la critique à l'endroit d'une élite blanche qui aurait volontairement retardé les secours, puis la reconstruction afin de " moderniser » La Nouvelle-Orléans, c'est-à-dire la débarrasser de ses éléments pathogènes, aux premiers rangs desquels les musiciens noirs. Trois personnages incarnent ce trope: le professeur d'université Creighton Bernette qui, lors d'un entretien filmé pour une télévision britannique, qualifie l'inefficacité de l'État fédéral à entretenir les digues de «putain de boulette aux proportions dantesques 25 »; le musicien DJ Davis qui, dans son rap « Shame, Shame, Shame », fustige le racisme de Georges W. Bush, Jr. (1.5) ; et le promoteur immobilier C.J. Ligori, dont la morale cynique (« on ne doit jamais gaspiller une catastrophe $\left.{ }^{26} »\right)$ est mise en acte par son associé, l'entrepreneur Nelson Hidalgo, qui rachète à bas prix des biens endommagés par l'inondation afin de spéculer sur leur valeur foncière à venir.

Treme s'en prend particulièrement au scandale des cités HLM (Calliope Projects, par exemple) maintenues fermées alors qu'elles ne furent pas touchées par l'inondation, mais aussi au fiasco bureaucratique que fut le programme « Road Home » (2.4), ou aux maigres versements des compagnies d'assurance qui assurent les dégâts causés par la tornade, et non par l'inondation. Les bavures policières sont également une préoccupation des auteurs, comme celle du Pont Danzinger, lorsque des policiers chargés de surveiller des détenus évacués d'une prison inondée commirent des meurtres, puis,

${ }^{24}$ Le sigle FEMA (Federal Emergency Management Agency, ou Agence fédérale pour la gestion des situations d'urgences) devient, graffé sur un mur, " Fix Everything, My Ass » littéralement « Réparez tout, mon cul » (générique).

25 « A fuck-up of epic proportions » (1.1)

26 « You should never let a disaster go away, as they say. » (2.1) 
lors des audiences, maquillèrent ces meurtres en cas de légitime défense ${ }^{27}$. Dans les saisons 2 et 3, l'enquête de l'avocate Toni Bernette sur le meurtre de Joey Abreu par un policier, quoique fictif, s'appuie indubitablement sur la perception très négative que les habitants défavorisés de La Nouvelle-Orléans ont eue de l'attitude de la police lors des inondations. Romain Huret, historien et auteur d'un livre remarquable sur le sujet des rapports entre Katrina, l'État américain et les pauvres, cite plusieurs témoignages allant dans ce sens :

L'incohérence des missions confiées aux sauveteurs de la garde nationale entraîne des réactions d'hostilité : «Nous n'avions ni nourriture, ni eau, mais ils essayaient d'arrêter les pillards [...], cela m'a sérieusement foutu les boules », se souvient un citoyen de la ville qui est resté enfermé dans le Superdome. " J'ai perdu tout respect pour la police », regrette pour sa part Keith Wagner, lorsqu'il décrit l'absence des forces de l'ordre dans les rues ${ }^{28}$.

Enfin, d'un point de vue méta-sériel, c'est-à-dire si l'on prend en compte la série dans l'espace des séries et ses rapports avec celui-ci, Treme offre tant d'éléments de continuum avec The Wire qu'il est difficile pour les téléspectateurs de ne pas s'attendre à une série sur la violence, la pauvreté et le racisme dans l'Amérique contemporaine. De quoi se compose cet horizon d'attente? Premièrement, Simon et Overmyer réengagèrent une demi-douzaine de comédiens présents dans leurs précédentes séries. Antoine Batiste est joué par Wendell Pierce, le fameux detective Bunk Moreland de The Wire, et Albert "Chief» Lambreaux est joué par Clarke Peters, le detective Lester Freamon de The Wire ${ }^{29}$. Deuxièmement, le thème de la corruption, si prégnant dans The Wire, n'est pas absent de Treme, par exemple à travers la pratique du homecooking (clientélisme dans l'attribution des contrats municipaux). Par exemple, Nelson Hidalgo cherche à récupérer les contrats d'entretien du réseau électrique en graissant la patte du councilman Oliver (2.10). Enfin, les bavures policières et la planification immobilière illicite constituent bel et bien des veines narratives de la série, à plus forte raison dans la saison 3.

Cela étant dit, la charge contestataire est moins lourde que dans The Wire, car le traitement est différent. Ainsi, dans certaines scènes, les spectateurs sont tentés de rire des personnages les plus militants. C'est le cas des deux personnages emblématiques de la critique à tout

\footnotetext{
27 «Police are Charged in Post-Katrina Shootings », The New York Times, 13 juillet 2010, cité dans Romain Huret, Katrina, 2005 : L'Ouragan, l'État et les pauvres aux États-Unis, Paris, École des Hautes Études en Sciences Sociales, 2010, p. 56.

28 Huret, p. 57, citations originelles dans Voices Rising : Stories from the Katrina Narrative Project, éd. Rebeca Antoine, New Orleans, UNO Press, 2008, p. 33 et p. 42.

${ }^{29}$ On retrouve également deux actrices qui jouaient dans Homicide: Life on the Street (NBC, 1993-1999) pour l'une et The Corner (HBO, 2000) pour l'autre : « Toni » Bernette est en effet jouée par Melissa Leo (la detective Kay Howard dans Homicide) et LaDonna Batiste-Williams est interprétée par Khandi Alexander (« Fran » Boyd dans The Corner).
} 
crin contre l'État fédéral que sont Creighton Bernette et DJ Davis. Ils sont quelque peu discrédités par les auteurs car leur militantisme à la fois convenu et passionnel est mis en scène de manière emphatique, voire grotesque. De plus, Treme n'est pas un documentaire à charge à la manière de When the Levees Broke (A Requiem in Four Acts) de Spike Lee (2010) ou de Trouble the Water de Tia Lessin (2008). Enfin, cette absence de didactisme provient d'un choix esthétique exprimé par l'auteur lui-même. Lors d'une conférence à Tulane University, David Simon répondit ainsi à un membre de l'auditoire qui lui demandait pourquoi Treme ne dénonçait pas plus frontalement la corruption :

Il est fort probable que les habitants (de La Nouvelle-Orléans) durent affronter des erreurs, $[. .$.$] en termes de gestion urbaine, semblables à$ celles de Baltimore; ces éléments dystopiques doivent être pris au sérieux ; [...] mais si vous concevez toute chose selon le désir de faire une série engagée politiquement, vos personnages deviennent des figures de papier sans épaisseur humaine ${ }^{30}$.

Le projet central reste donc de discuter la place de la culture dans la reconstruction et le maintien en vie de populations affaiblies et délaissées.

\section{Conclusion}

La reprise dans Treme est donc multiforme. Dans sa version musicale (cover), elle est tout à la fois hommage aux classiques, éducation, terreau nécessaire et inconscient, source de création et d'hybridation. Entendue comme renaissance ou renouveau (recover), elle est au cœur des vies des personnages, mais elle n'est jamais simple ou acquise. La série avance à petits pas, comme un Mardi Gras Indian qui coud son costume point après point. Enfin, dans ses relations critiques avec l'en-dehors du récit (uncover), le motif de la reprise fait de Treme une œuvre qui n'aurait pas la prétention de tout expliquer, mais conserverait en son cour la préoccupation de transcrire fidèlement un moment de l'histoire américaine, ainsi que l'expérience des gens de peu. Ce regard non surplombant permet une critique sociopolitique qui se situe aux antipodes d'un certain dogmatisme présent dans des documentaires dits engagés.

Ces trois motifs de la reprise fonctionnent en synergie et s'enchevêtrent pour donner à la série une tonalité idiosyncratique difficile à décrire. Mélange de lenteur, d'absence d'action, de jouissance du temps, de compassion et d'indignation, cette «note Treme», comme on parle de « blue note » dans le blues, trouve son origine dans

$3^{0}$ «Inhabitants might have to confront certain civic flaws and dynamics, like in Baltimore; these dystopic elements have to be addressed [...] but if you frame everything to the desire of making a political statement, your characters become cardboard. " Simon, " The Art of Treme », échange avec le public, op. cit. 
le recours généralisé à la figure de la reprise. À cet égard, je fais miens les propos du philosophe Mathieu Potte-Bonneville, qui s'exprimait ainsi sur les rapports des séries contemporaines au monde moderne :

La série est fondée sur la répétition, avec les mêmes personnages, les mêmes génériques, les mêmes débuts. Il y a là l'expression d'un piétinement qui définit notre monde voué à la répétition, privé d'avenir, de projets politiques, d'utopies. Sauf que, grâce à l'emboîtement épisode/saison/série, la répétition du quotidien se nuance de variations et un récit se trame malgré tout. La série prend en charge la question $[\ldots]$ : qu'est-ce qui change quand rien ne change ${ }^{31}$ ?

Treme se nourrit de cette spécificité du genre sériel tout en l'enrichissant. En mettant la reprise, entendue sous ses trois acceptions, au cœur de sa facture autant au niveau de la diégèse que de la métadiégèse, elle ne cesse d'interroger les rapports complexes entre le pouvoir et la culture. Il s'agit bien d'une interrogation, car les parcours complexes des personnages empêchent de conclure à une hypothétique victoire certaine et durable de la culture sur les forces adversaires. Il en va de même pour les vrais habitants de La NouvelleOrléans : si certains musiciens soulignent que leur culture a survécu, notamment grâce au rayonnement d'une œuvre comme Treme à travers le monde ${ }^{2}$, il faut souligner, pour conclure, que cette survie est le fruit d'un processus lent et fragile. Comme l'explique Simon luimême : «La Nouvelle-Orléans est revenue à la vie [...], non pas grâce à une volonté politique ou à une quelconque directive économique, mais grâce à la culture [...] : un défilé après l'autre, un solo de trombone après l'autre, un plat de crevettes à l'étouffée après l'autre, une parade costumée après l'autre 33 . »

\section{Bibliographie}

ANTOINE Rebeca (éd.), Voices Rising: Stories from the Katrina Narrative Project, New Orleans (LA), UNO Press, 2008.

COURTNEY George, « Keeping It "Reals" Narratives of New Orleans Jazz History as Represented in HBO's Treme », Television \& New Media, vol. 13, 2012., p. 225-234.

${ }^{31}$ Entretien collectif, "Ce que disent les séries télé », Le Nouvel Observateur, $\mathrm{n}^{\circ}$ 2476, 19 avril 2012, p. 106-109 (je souligne).

${ }^{2}$ Témoignage du musicien Big Sam Williams, « Music Brings a City Back: New Orleans and Treme", entretien vidéo pour San Francisco Classical Voice, 23 juin 2011, http://www.sfcv.org, consulté le 20/12/12.

33 « New Orleans has come back to the extent that it has come back not by political will, or economic fiat, but by culture [...] one second line, trombone solo, étouffée, Indian suited parade at a time », Simon, " The Art of Treme », op. cit. 
CRUTCher Michael Eugene, Tremé: Race and Place in a New Orleans Neighborhood, Athens (GA), University of Georgia Press, 2010.

Dessinges Catherine, Dominique Gendrin, Wendy HAJJAR, « Fiction and Reality in HBO's Treme: A Narrative Alchemy at the Service of Political Truth », TV/Series, $\mathrm{n}^{\circ} 1$, juin 2012 (Les Séries télévisées américaines contemporaines : Entre la fiction, les faits, et le réel, éd. Ariane Hudelet et Sophie Vasset) : http://www.univlehavre.fr/ulh services/IMG/pdf/11-Dessinges et al-.pdf

Entretien collectif, "Ce que disent les séries télé », Le Nouvel Observateur, $\mathrm{n}^{\circ}$ 2476, 19 avril 2012, p. 106-109.

FrANkLIN Sara B., "Tradition, Treme, and the New Orleans Renaissance: an interview with Lolis Eric Elie », Southern Cultures, vol. 18, été 2012, p. 32-44.

HAVRILESKY Heather, «David Simon's Magnificent, Melancholy: Treme », Salon, 4 avril 2010.

Herman Gray, «Recovered, Reinvented, Reimagined Treme, Television Studies and Writing New Orleans », Television \& New Media, vol. 13, 2012, p. 268-278.

HURET Romain, Katrina, 2005 : L'Ouragan, l'État et les pauvres aux États-Unis, Paris, École des Hautes Études en Sciences Sociales, 2010.

LEFREBVRE Hugo, Enjeux de la reconstruction de la Nouvelle-Orléans après l'ouragan Katrina, Éditions L’Harmattan, 2008.

Patterson Troy, « Treme », Slate, 9 avril 2010.

SeIDman Karl F., Coming Home to New Orleans: Neighborhood Rebuilding After Katrina, Oxford University Press, 2013.

SÉRISIER Pierre, "Treme - l'espoir à fleur de ville », Le Monde des Séries, 3 décembre 2012,

http://seriestv.blog.lemonde.fr/2012/12/o3/treme-lespoir-a-fleur-deville/

Stanley Alessandra, « After Katrina, Staying Afloat With Music », The New York Times, 9 avril 2010.

WALKer Dave, "Treme: Explained », The Times-Picayune Online, http://www.nola.com/treme-hbo/. 


\section{L'auteur}

Peter Marquis est agrégé d'anglais, docteur en histoire et civilisations de l'École des Hautes Études en Sciences Sociales, Maître de conférences en études américaines à l'Université de Rouen, et chercheur rattaché à l'ERIAC (Université de Rouen) et au Centre d'Études Nord-Américaines (CENA/MASCIPO, UMR 8160). Ses recherches actuelles portent sur l'histoire des loisirs, la culture populaire américaine (sport, télévision, musique) et le traitement de la parentalité dans les médias. Pour en savoir plus : http://univrouen.academia.edu/PeterMarquis. 